\title{
Validated Enclosure of Uncertain Nonlinear Equations Using SIVIA Monte Carlo
}

\author{
Nisha Rani Mahato, Luc Jaulin (D), S. Chakraverty (i) and Jean Dezert
}

\begin{abstract}
The dynamical systems in various science and engineering problems are often governed by nonlinear equations (differential equations). Due to insufficiency and incompleteness of system information, the parameters in such equations may have uncertainty. Interval analysis serves as an efficient tool for handling uncertainties in terms of closed intervals. One of the major problems with interval analysis is handling "dependency problems" for computation of the tightest range of solution enclosure or exact enclosure. Such dependency problems are often observed while dealing with complex nonlinear equations. In this regard, initially, two test problems comprising interval nonlinear equations are considered. The Set Inversion via Interval Analysis (SIVIA) along with the Monte Carlo approach is used to compute the exact enclosure of the test problems. Further, the efficiency of the proposed approach has also been verified for solving nonlinear differential equation (Van der Pol oscillator) subject to interval initial conditions.
\end{abstract}

Keywords Uncertain nonlinear equations - Nonlinear oscillator - Dependency problem $\cdot$ SIVIA Monte Carlo $\cdot$ Contractor

\footnotetext{
N. R. Mahato $(\bowtie) \cdot S$. Chakraverty

Department of Mathematics, National Institute of Technology Rourkela, Rourkela 769008, Odisha, India

e-mail: nisha.mahato1@gmail.com

S. Chakraverty

e-mail: sne_chak@yahoo.com

L. Jaulin

ENSTA-Bretagne, Lab-STICC, CNRS 6285, 2 rue François Verny, 29806 Brest, France

e-mail: lucjaulin@gmail.com

J. Dezert

The French Aerospace Lab, 91120 Palaiseau, France

e-mail: jean.dezert@onera.fr
} 


\section{Introduction}

Various vibration problems in science and engineering disciplines, viz., structural mechanics, control theory, seismology, physics, and biology may be expressed in terms of nonlinear equations, system of nonlinear equations, and nonlinear differential equations. Generally, the parameters in such equations deal with precise variables. But, the insufficiency and incompleteness of the system information often led to parameters or variables with imprecision or uncertainty. For instance, let us consider a nonlinear damped spring-mass system as given in Fig. 1 governed by the equation,

$$
m \ddot{x}+c \dot{x}+\alpha \dot{x}^{2}+k x+\beta x^{3}=f(t)
$$

where $m, c$, and $k$ are respectively mass, damping, and stiffness of the nonlinear system. Here, the external force applied on the system is $f(t)$ with damping force $f_{d}=c \dot{x}+\alpha \dot{x}^{2}$ and spring force $f_{s}=k x+\beta x^{3}$.

The uncertainty of the material properties in Eq. (1) led to the uncertain nonlinear differential equation. Such uncertainties may be modeled either using probabilistic approach, interval computation or fuzzy set theory. In case of nonavailability of sufficient experimental data, probabilistic methods may not be able to deliver reliable results. Moreover, in fuzzy set theory, a fuzzy number is expressed in terms of closed intervals through the $\alpha$-cut approach. As such, interval analysis has emerged as a powerful tool for various practical problems in handling the uncertainties.

In the early 1960 s, the pioneer concept related to interval computations, functions, matrices, integral, and differential equations has been started by Moore [12-14]. System of equations, algebraic eigenvalue problems, and second-order initial and boundary value problems have been discussed by Alefeld and Herzberger [2]. Guaranteed interval computations with respect to set approximations, parameter, and state estimation with applications in robust control and robotics are addressed by Jaulin et al. [10]. While dealing with interval computations, one of the major obstacles is to handle the "dependency problems" effectively such that the tightest enclosure of solution bound may be obtained. Such dependency problems often occur in dealing with systems governed by complex nonlinear equations which lead to overestimation of solution bounds. The dependency problem due to overestimation (wrapping effect) has been studied by Krämer [11] with respect to generalized interval arithmetic proposed by Hansen [9]. The other approach for the reduction of overestima-

Fig. 1 Damped spring-mass system

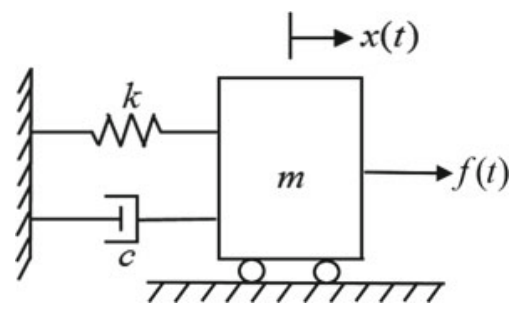


tion while handling dependency problem may be performed using contractors [10], affine arithmetic [16], and/or parametric forms. As such, the present work proceeds with the introduction section. The preliminaries of classical arithmetic of interval analysis (IA) along with its application for two complex nonlinear equations comprising imprecise variables are considered in Sect. 2. The Set Inversion via Interval Analysis (SIVIA) along with the Monte Carlo approach is then used to compute the exact enclosure of the two test problems in Sect.3. Further, the proposed approach has also been verified for computing validated enclosure of nonlinear differential equation (Van der Pol oscillator) subject to interval initial conditions in Sect. 4.

\section{Classical Interval Computations}

Interval analysis deals with interval computations on a set of closed intervals $\mathbb{I R}$ of real line $\mathbb{R}$, in order to obtain the tightest bound or enclosure for uncertain systems. A closed interval $[x] \in \mathbb{I}$ is denoted by $[x]=[\underline{x}, \bar{x}]$ such that

$$
[x]=[\underline{x}, \bar{x}]=\{t \mid \underline{x} \leq t \leq \bar{x} \text {, where } \underline{x}, \bar{x} \in \mathbb{R}\} .
$$

Here, $\underline{x}=\inf [x]$ is the inifimum or lower bound of $[x]$ and $\bar{x}=\sup [x]$ is the supremum or upper bound of $[x]$. The width and center of $[x]$ may be referred to as $[x]^{w}=\bar{x}-\underline{x}$ and $[x]^{c}=\frac{\underline{x}+\bar{x}}{2}$, respectively.

Basic operations using classical interval arithmetic given in Moore et al. [14] are illustrated as follows:

- Addition: $[x]+[y]=[\underline{x}+\underline{y}, \bar{x}+\bar{y}]$

- Subtraction: $[x]-[y]=[\underline{x}-\bar{y}, \bar{x}-y]$

- Multiplication: $[x] \cdot[y]=[\min \{S .([x],[y])\}, \max \{S .([x],[y])\}]$, where $S .([x],[y])=\{\underline{x} y, \underline{x} \bar{y}, \bar{x} y, \overline{x y}\}$

- Division: $[x] /[y]=\left\{\begin{array}{cc}{[\underline{x}, \bar{x}] \cdot\left[\frac{1}{\bar{y}}, \frac{1}{y}\right],} & 0 \notin[\underline{y}, \bar{y}], \\ (-\infty, \infty), & 0 \in[\underline{y}, \bar{y}]\end{array}\right.$

\section{- Power:}

- If $n>0$ is an odd number, then $[x]^{n}=\left[\underline{x}^{n}, \bar{x}^{n}\right]$

- If $n>0$ is an even number, then $[x]^{n}=\left\{\begin{array}{cc}{\left[\underline{x}^{n}, \bar{x}^{n}\right],} & {[x]>0} \\ \left.\bar{x}^{n}, \underline{x}^{n}\right], & {[x]<0} \\ {\left[0, \max \left\{\underline{x}^{n}, \bar{x}^{n}\right\}\right],} & 0 \in[x]\end{array}\right.$

Then, we have illustrated two test examples for the implementation of basic interval arithmetic in Examples 1 and 2.

Example 1 Compute the bound $\left[z_{1}\right]$ satisfying constraint

$$
z_{1}=x_{1} y_{1}+x_{1} y_{3}+x_{3} y_{1}
$$


such that $x_{1}+x_{2}+x_{3}=1$ and $y_{1}+y_{2}+y_{3}=1$. Here, $x_{1} \in\left[x_{1}\right]=[0.2,0.3], x_{2} \in$ $\left[x_{2}\right]=[0.1,0.2], y_{1} \in\left[y_{1}\right]=[0.4,0.6]$, and $y_{2} \in\left[y_{2}\right]=[0.2,0.3]$.

Using classical IA, the bounds $\left[x_{3}\right]$ and $\left[y_{3}\right]$ are initially estimated as

$$
\left[x_{3}\right] \sim 1-\left[x_{1}\right]-\left[x_{2}\right]=[0.5,0.7] \text { and }\left[y_{3}\right] \sim 1-\left[y_{1}\right]-\left[y_{2}\right]=[0.1,0.4]
$$

respectively with respect to the constraints $x_{1}+x_{2}+x_{3}=1$ and $y_{1}+y_{2}+y_{3}=1$. Then, the bound $\left[z_{1}\right]$ is obtained as

$$
\left[z_{1}\right]^{\mathbf{I A}} \sim\left[x_{1}\right] \cdot\left[y_{1}\right]+\left[x_{1}\right] \cdot\left[y_{3}\right]+\left[x_{3}\right] \cdot\left[y_{1}\right]=[0.30,0.72] .
$$

Further, we have considered a more complicated nonlinear constraint in Example 2, related to problems of multi-criteria decision-making under imprecise scores given in Dezert et al. [7].

Example 2 [7] Compute the bound $\left[z_{2}\right]$ satisfying constraint

$$
z_{2}=z_{1}+\frac{x_{1}^{2} y_{2}}{x_{1}+y_{2}}+\frac{y_{1}^{2} x_{2}}{y_{1}+x_{2}}
$$

such that $x_{1} \in[0.2,0.3], x_{2} \in[0.1,0.2], y_{1} \in[0.4,0.6]$, and $y_{2} \in[0.2,0.3]$.

Here, the bound of $\left[z_{2}\right]$ is obtained as

$$
\left[z_{2}\right]^{\mathbf{I A}} \sim\left[z_{1}\right]^{\mathbf{I A}}+\frac{\left[x_{1}\right]^{2}\left[y_{2}\right]}{\left[x_{1}\right]+\left[y_{2}\right]}+\frac{\left[y_{1}\right]^{2}\left[x_{2}\right]}{\left[y_{1}\right]+\left[x_{2}\right]}=[0.3333,0.9315] .
$$

The enclosures obtained in Eqs. (3) and (5) have been compared with enclosures obtained using the Monte Carlo simulation in Table 1.

Here, the Monte Carlo simulation approach using uniformly distributed 100,000 independent random sample values of variables $x_{1}, x_{2}, y_{1}$, and $y_{2}$ have been considered, where $x_{1} \sim U\left(\left[x_{1}\right]\right), x_{2} \sim U\left(\left[x_{2}\right]\right), y_{1} \sim U\left(\left[y_{1}\right]\right)$, and $y_{2} \sim U\left(\left[y_{2}\right]\right)$. From Table 1, it is worth mentioning that the bounds for $i=1,2$ satisfy

$$
\left[z_{i}\right]^{\mathbf{M C}} \subset\left[z_{i}\right]^{\mathbf{I A}}
$$

In case of more sample values, the Monte Carlo simulation may yield better interval enclosure with respect to the constraints (2) and (4), but such approach is inefficient with respect to computational time. So, we may consider the problem in handling

Table 1 Interval bounds of $z_{1}$ and $z_{2}$

\begin{tabular}{l|l|l}
\hline \multirow{2}{*}{$i$} & \multicolumn{2}{|c}{ Interval bounds } \\
\cline { 2 - 3 } & {$\left[z_{i}\right]^{\mathbf{I A}}$} & {$\left[z_{i}\right]^{\mathbf{M C}}$} \\
\hline 1 & {$[0.30,0.72]$} & {$[0.3850,0.5935]$} \\
2 & {$[0.3333,0.9315]$} & {$[0.4617,0.6825]$} \\
\hline
\end{tabular}


interval computations as to interpret the tightest or the exact enclosure $\left[z_{i}\right]$ of $z_{i}$ that satisfies

$$
\left[z_{i}\right]^{\mathbf{M C}} \subset\left[z_{i}\right] \subset\left[z_{i}\right]^{\mathbf{I A}}
$$

such that

$$
\begin{gathered}
\inf \left[z_{i}\right]^{\mathbf{I A}} \leq \inf \left[z_{i}\right] \leq \inf \left[z_{i}\right]^{\mathbf{M C}} \text { and } \sup \left[z_{i}\right]^{\mathbf{M C}} \leq \sup \left[z_{i}\right] \leq \sup \left[z_{i}\right]^{\mathbf{I A}} \\
\text { or } \underline{z}_{i}^{\mathbf{I A}} \leq \underline{z}_{i} \leq \underline{z}_{i}^{\mathbf{M C}} \text { and } \bar{z}_{i}^{\mathbf{M C}} \leq \bar{z}_{i} \leq \bar{z}_{i}^{\mathbf{I A}} .
\end{gathered}
$$

Although in the above computations, the interval arithmetic looks simple for basic operations with intervals and seems appealing, the "dependency problem" is a major obstacle when complicated expressions have to be computed in order to find the tightest enclosure. In this regard, the dependency effect has been discussed in detail in the next section.

\subsection{Dependency Problem in IA}

Variable or parameter dependency problem in IA is generally exhibited when we have more than one occurrence of imprecise parameter in the governing constraint. For instance, in case of the nonlinear constraint

$$
z=x^{2}+y^{2} \text { for } x \in[0.1,0.5] \text { and } y \in[-0.6,0.1]
$$

the occurrence of each imprecise variable $x$ and $y$ is once. The computation of enclosure with respect to constraint $z=x^{2}+y^{2}$ using classical IA results in $[z]^{\mathbf{I A}}=[0.01,0.61]$ which is found equivalent to the Monte Carlo simulation of $x \sim U([0.1,0.5]), y \sim U([-0.6,0.1])$ for 100,000 sample values that yields $[z]^{\mathbf{M C}}=[0.01,0.61]$. But, the complexity occurs while dealing with complex nonlinear constraints as given in Examples 1 and 2, where the dependency effect is exhibited due to multiple occurrences of imprecise variables.

The dependency effect may be reduced by replacing the constraint given in Eq. (2) with an equivalent simpler constraint having less (or none) redundant variables. For instance, the equivalent constraint

$$
z_{1}=\left(1-x_{2}\right) y_{2}+x_{3} y_{1}
$$

results in a better enclosure approximation $\left[z_{1}\right]^{\mathbf{I A}}=[0.34,0.66]$. Here, the interval bound $[0.34,0.66]$ is contained in the bound $[0.30,0.72]$ obtained using the equivalent constraint given in Eq. (2). But, on the other hand, an equivalent constraint

$$
z_{1}=\left(1-y_{2}\right) x_{1}+\left(1-x_{2}\right) y_{1}-x_{1} y_{1}
$$


results in an overestimated bound $\left[z_{1}\right]^{\mathbf{I A}}=[0.28,0.70]$. Due to such dependency, the interval bounds often yield overestimation of the tightest enclosure. Similar, dependency effect is exhibited while computing $\left[z_{2}\right]^{\mathbf{I A}}$ for constraints $z_{2}=z_{1}+$ $\left(\frac{1}{x_{1} y_{2}}+\frac{1}{x_{1}^{2}}\right)^{-1}+\frac{y_{1}^{2} x_{2}}{y_{1}+x_{2}}$ and $z_{2}=z_{1}+\frac{x_{1}^{2} y_{2}}{x_{1}+y_{2}}+\left(\frac{1}{y_{1} x_{2}}+\frac{1}{y_{1}^{2}}\right)^{-1}$ with respect to (4). As such, identification of constraint yielding the tightest enclosure is cumbersome. In this regard, the problem formulation for reduction of overestimation due to dependency effect has been carried out in the next section.

\subsubsection{Problem Formulation}

The main aim in the present work is to compute the tightest enclosure $\left[\underline{z}_{i}, \bar{z}_{i}\right]$ or exact enclosure such that $\left[z_{i}\right]^{\mathbf{M C}} \sim\left[z_{i}\right]^{\mathbf{I A}}$ or

$$
\underline{z}_{i}^{\mathbf{I A}}=\underline{z}_{i}=\underline{z}_{i}^{\mathbf{M C}} \text { and } \bar{z}_{i}^{\mathbf{M C}}=\bar{z}_{i}=\bar{z}_{i}^{\mathbf{I A}} \text {. }
$$

associated with some nonlinear constraint $z_{i}=f\left(x_{1}, x_{2}, y_{1}, y_{2}\right)$, where $x_{i} \in\left[x_{i}\right]$ and $y_{i} \in\left[y_{i}\right]$ for $i=1,2$. In this regard, the SIVIA Monte Carlo approach based on the set inversion using interval computations and the Monte Carlo simulation has been proposed to estimate exact bounds in the next section.

\section{SIVIA Monte Carlo Approach}

Initially, the general procedure of SIVIA has been incorporated in Sect. 3.1 followed by contractors in Sect. 3.2. Finally, the combination of SIVIA with the Monte Carlo approach has been performed in Sect.3.3.

\subsection{SIVIA}

Set inversion of a typical set $\mathbb{X} \subset \mathbb{R}^{m}$ with respect to function $f: \mathbb{R}^{m} \rightarrow \mathbb{R}^{n}$ is expressed as

$$
\mathbb{X}=f^{-1}(\mathbb{Y})=\left\{\mathbf{x} \in \mathbb{R}^{m} \mid f(\mathbf{x}) \in \mathbb{Y}\right\}
$$

where $\mathbb{Y} \subset \mathbb{R}^{n}$. In case of SIVIA [10], an initial search set [ $\left.\mathbf{x}_{0}\right]$ is assumed containing the required set $\mathbb{X}$. Then, using sub-pavings as given in Fig. 2, the desired enclosure of solution set $\mathbb{X}$ is obtained based on the inclusion properties:

1. Case $I:[f]([\mathbf{x}]) \subset \mathbb{Y} \Longrightarrow[\mathbf{x}] \subset \mathbb{X}$, then $[\mathbf{x}]$ is a solution,

2. Case $I I:[f]([\mathbf{x}]) \cap \mathbb{Y}=\phi \Longrightarrow[\mathbf{x}] \cap \mathbb{X}=\phi$, then $[\mathbf{x}]$ is not a solution,

3. Case III: $[f]([\mathbf{x}]) \cap \mathbb{Y} \neq \phi$ and $[f]([\mathbf{x}]) \not \subset \mathbb{Y}$ then, $[\mathbf{x}]$ is an undetermined solution. 


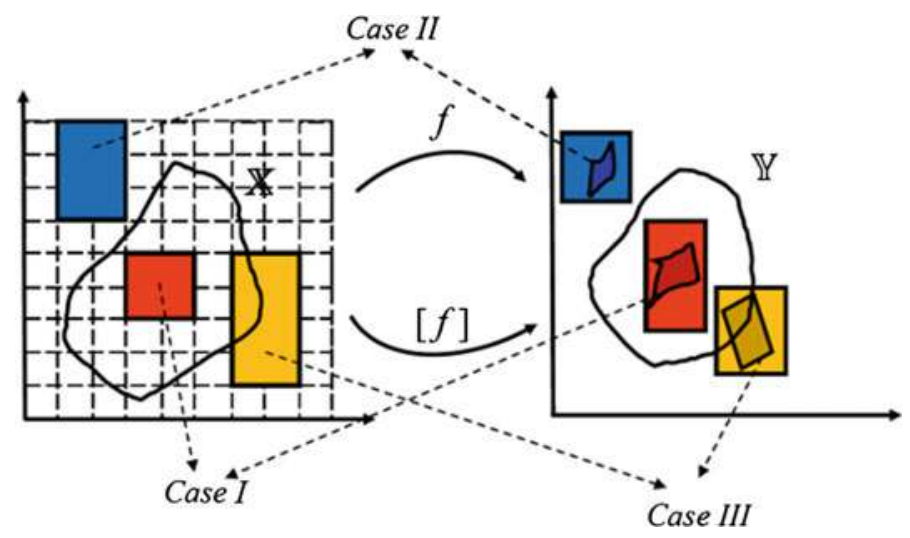

Fig. 2 Set Inversion via Interval Analysis

The detailed illustration of set computation using SIVIA based on regular subpavings, bisections, etc., may be found in [10]. The sub-pavings in SIVIA may be improved with the usage of contractors discussed in the next section.

\subsection{Contractor}

Contractor: $[3,10]$ A contractor $\mathcal{C}$ associated with a set $\mathbb{X} \subset \mathbb{R}^{n}$ over domain $\mathbb{D}$ is an operator

$$
\mathcal{C}: \mathbb{I} \mathbb{R}^{n} \rightarrow \mathbb{I R}^{n}
$$

satisfying the following properties:

- Contraction: $\mathcal{C}([\mathbf{x}]) \subset[\mathbf{x}], \forall[\mathbf{x}] \in \mathbb{I} \mathbb{R}^{n}$,

- Completeness: $\mathcal{C}([\mathbf{x}]) \cap \mathbb{X}=[\mathbf{x}] \cap \mathbb{X}, \forall[\mathbf{x}] \in \mathbb{R}^{n}$.

The pictorial representation of implementation of a contractor over the set $\mathbb{X} \subset \mathbb{R}^{2}$ is illustrated in Fig. 3.

There exist various types of contractors, viz., fixed-point, forward-backward, Newton, and Gauss-Seidel contractors. Contractor based set inversion of leminscate

Fig. 3 Contraction of $[x]$

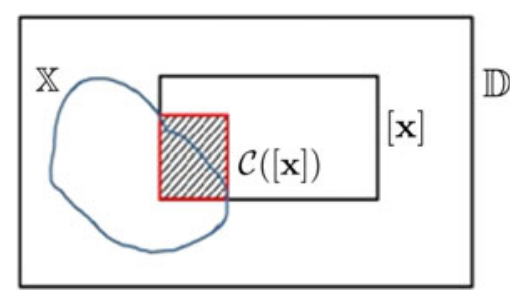


Fig. 4 SIVIA of leminscate curve with width $[2,3]$

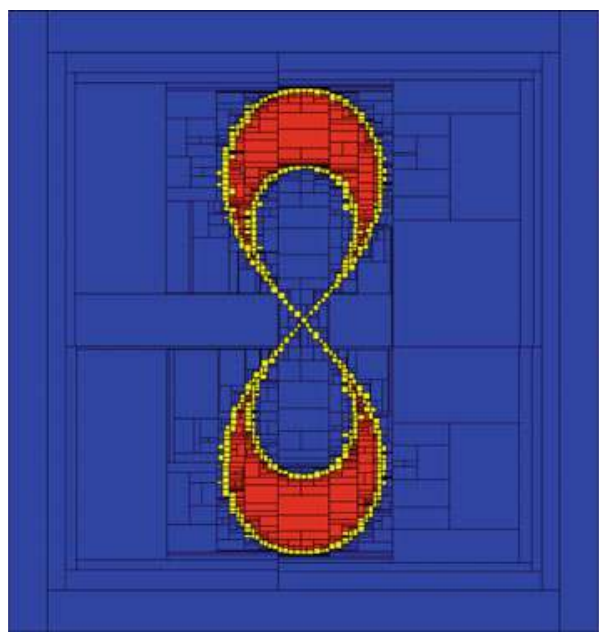

curve $\left(x^{2}+y^{2}\right)^{2}+a^{2}\left(x^{2}-y^{2}\right)=0$ having width $a \in[2,3]$ has been obtained based on the PyIbex library [6] and depicted in Fig. 4, where the initial search set is $[-4,4] \times[-4,4]$.

In order to perform the SIVIA Monte Carlo approach, we have used forwardbackward and fixed-point contractors. Detailed implementation of forward-backward and fixed-point contractors have been incorporated in the Appendix.

\subsection{SIVIA Monte Carlo}

SIVIA Monte Carlo is a two-form iterative methodology that includes implementation of SIVIA using contractor programming and the Monte Carlo simulation till the exact enclosure is obtained satisfying (11). In this regard, the iterative procedure is incorporated in Algorithm 1 with respect to constraint $z=f\left(x_{1}, x_{2}, \ldots, x_{n}\right)$ such that each $x_{i} \in\left[x_{i}\right] \in \mathbb{I}$ for $i=1,2, \ldots, n$. Here, the initial search set containing the exact enclosure is assumed as $\left[z_{0}\right]$.

Algorithm 1: Implementation of SIVIA Monte Carlo approach

Input: $\left[x_{i}\right]$ for $i=1,2, \ldots, n$; Initial domain $[\mathbf{x}]=\left\{\left[x_{1}\right],\left[x_{2}\right], \ldots,\left[x_{n}\right]\right\} \in \mathbb{D}$; Initial search set $\left[z_{0}\right]$

Step 1: Compute enclosure using Monte Carlo

$$
\underline{z}^{\mathbf{M C}}=m c l([\mathbf{x}]) \text { and } \bar{z}^{\mathbf{M C}}=m c u([\mathbf{x}])
$$

Step 2: Compute enclosure using contractors

$$
\underline{z}^{\mathbf{I A}}=\operatorname{Ctcl}\left([\mathbf{x}],\left[z_{0}\right]\right) \text { and } \bar{z}^{\mathbf{I A}}=C t c u\left([\mathbf{x}],\left[z_{0}\right]\right)
$$

Step 3: Improve lower and upper range of $z$ 


$$
\underline{z} \in\left[\underline{z}^{\mathbf{I A}}, \underline{z}^{\mathbf{M C}}\right] \text { and } \bar{z} \in\left[\bar{z}^{\mathbf{M C}}, \bar{z}^{\mathbf{I A}}\right]
$$

Step 4: Compute improved lower $[\underline{\mathbf{x}}]=[f]^{-1}\left(\left[\underline{z}^{\mathbf{I A}}, \underline{z}^{\mathbf{M C}}\right]\right)$ and upper $[\overline{\mathbf{x}}]=[f]^{-1}\left(\left[\bar{z}^{\mathbf{M C}}, \bar{z}^{\mathbf{I A}}\right]\right)$ domains using SIVIA

$[\underline{\mathbf{x}}],[\overline{\mathbf{x}}]=\operatorname{SIVIA}\left([\mathbf{x}],[f],\left[z_{0}\right], \epsilon\right)$

Step 5: Repeat steps 1 to 3 for domains $[\underline{\mathbf{x}}]$ and $[\overline{\mathbf{x}}]$

Step 6: Repeat step 4 for different domains $[\mathbf{x}]$ and $[\overline{\mathbf{x}}]$

Step 7: Iterate steps 4 and 5 till $\underline{z}=\underline{z}^{\mathbf{I A}} \sim \underline{z}^{\mathbf{M C}}$ and $\bar{z}=\bar{z}^{\mathbf{M C}} \sim \bar{z}^{\mathbf{I A}}$

Output: $[\underline{z}, \bar{z}]$

In Algorithm 1, $\operatorname{mcl}(\cdot), \operatorname{mcu}(\cdot)$ are functions that compute the minimum and maximum function values with respect to domain $[\mathbf{x}] \in \mathbb{D}$. Then, $\operatorname{Ctcl}(\cdot), \operatorname{Ctcu}(\cdot)$ uses forward-backward contractor along with fixed-point contractor for computing interval enclosure based on classical IA. Further, SIVIA $(\cdot)$ computes the set inversion for domain $[\mathbf{x}] \in \mathbb{D}$ based on constraint function $f$ with precision $\epsilon$.

Let us again consider the Examples 1 and 2 in order to compute the exact enclosure using the SIVIA Monte Carlo in Example 3.

Example 3 Compute the interval bounds for the constraints

$$
z_{1}=x_{1} y_{1}+x_{1} y_{3}+x_{3} y_{1} \text { and } z_{2}=z_{1}+\frac{x_{1}^{2} y_{2}}{x_{1}+y_{2}}+\frac{y_{1}^{2} x_{2}}{y_{1}+x_{2}}
$$

using the SIVIA Monte Carlo such that $x_{1}+x_{2}+x_{3}=1$ and $y_{1}+y_{2}+y_{3}=$ 1. Again, $x_{1} \in\left[x_{1}\right]=[0.2,0.3], x_{2} \in\left[x_{2}\right]=[0.1,0.2], y_{1} \in\left[y_{1}\right]=[0.4,0.6]$, and $y_{2} \in\left[y_{2}\right]=[0.2,0.3]$. Using Algorithm 1 for SIVIA precision $\epsilon=0.001$ and different sample values, viz., 100,000, 1000, 100, 10, the tightest enclosures with respect to constraints $z_{1}=x_{1} y_{1}+x_{1} y_{3}+x_{3} y_{1}$ and $z_{2}=z_{1}+\frac{x_{1}^{2} y_{2}}{x_{1}+y_{2}}+\frac{y_{1}^{2} x_{2}}{y_{1}+x_{2}}$ for different sample values are obtained and incorporated in Tables 2 and 3 , respectively.

It may be observed from Table 2 that the SIVIA Monte Carlo method iteratively converges to the exact enclosure [0.38,0.6] (up to two decimals) even for fewer sample values, viz., 100 and 10. Also, it may be noted that the iterative enclosures converge to exact bound though the computational time increases from 5.1388 to $9.511 \mathrm{~s}$ for different samples ranging from 100,000 to 10, respectively. From Table 2, the proposed method seems appealing as even for fewer sample values the convergent or exact solution bound is achieved. Many practical application problems do not yield sufficient data and sometimes the availability of large data is cost effective, in such cases, the proposed method may be used to obtain exact enclosure and the increase in the computational time may be neglected.

Similar observations of exact enclosure convergence may be found in Table 3 with respect to different sample values. Moreover, due to the complexity of the constraint (4), the required computational time $24.847 \mathrm{~s}$ for $\left[z_{2}\right]$ is comparatively higher than time $9.511 \mathrm{~s}$ required for $\left[z_{1}\right]$. Further, a nonlinear differential equation with respect to dynamic problems has been considered in the next section for verification and effectiveness of the SIVIA Monte Carlo approach. 
Table 2 Interval enclosure of $z_{1}$

\begin{tabular}{|c|c|c|c|c|}
\hline \multirow[t]{3}{*}{ Iterations } & \multicolumn{4}{|c|}{ SIVIA (0.001 precision) and Monte Carlo samples } \\
\hline & \multicolumn{2}{|l|}{100,000 samples } & \multicolumn{2}{|l|}{1000 samples } \\
\hline & $\underline{z}_{1} \in$ & $\bar{z}_{1} \in$ & $\underline{z}_{1} \in$ & $\bar{z}_{1} \in$ \\
\hline 1 & {$[0.3796,0.385]$} & {$[0.5935,0.6007]$} & {$[0.3796,0.3850]$} & {$[0.5935,0.6007]$} \\
\hline 2 & {$[0.3796,0.3807]$} & {$[0.5993,0.6007]$} & {$[0.3796,0.3822]$} & {$[0.5971,0.6007]$} \\
\hline 3 & {$[0.3796,0.3801]$} & {$[0.5999,0.6006]$} & {$[0.3796,0.3808]$} & {$[0.5987,0.6008]$} \\
\hline 4 & - & - & {$[0.3797,0.3803]$} & {$[0.5995,0.6007]$} \\
\hline$\left[z_{1}\right]$ & \multicolumn{2}{|c|}{$[0.38,0.6]$} & \multicolumn{2}{|c|}{$[0.38,0.6]$} \\
\hline Time (s) & \multicolumn{2}{|l|}{5.1388} & \multicolumn{2}{|l|}{5.5936} \\
\hline \multirow[t]{2}{*}{ Iterations } & \multicolumn{2}{|l|}{100 samples } & \multicolumn{2}{|l|}{10 samples } \\
\hline & $\underline{z}_{1} \in$ & $\bar{z}_{1} \in$ & $\underline{z}_{1} \in$ & $\bar{z}_{1} \in$ \\
\hline 1 & {$[0.3796,0.385]$} & {$[0.5935,0.6007]$} & {$[0.3796,0.385]$} & {$[0.5935,0.6007]$} \\
\hline 2 & {$[0.3796,0.3833]$} & {$[0.5965,0.6007]$} & {$[0.3796,0.384]$} & {$[0.5945,0.6007]$} \\
\hline 3 & {$[0.3797,0.3817]$} & {$[0.5982,0.6007]$} & {$[0.3796,0.3836]$} & {$[0.595,0.6007]$} \\
\hline 4 & {$[0.3797,0.3814]$} & {$[0.5989,0.6007]$} & {$[0.3797,0.3829]$} & {$[0.5971,0.6007]$} \\
\hline 5 & {$[0.3797,0.3808]$} & {$[0.5995,0.6006]$} & {$[0.3797,0.3811]$} & {$[0.5977,0.6007]$} \\
\hline 6 & {$[0.3797,0.3805]$} & {$[0.5996,0.6006]$} & {$[0.3797,0.3808]$} & {$[0.5978,0.6006]$} \\
\hline 7 & - & - & {$[0.3797,0.3805]$} & {$[0.5988,0.6006]$} \\
\hline$\left[z_{1}\right]$ & \multicolumn{2}{|c|}{$[0.38,0.6]$} & \multicolumn{2}{|c|}{$[0.38,0.6]$} \\
\hline Time (s) & \multicolumn{2}{|c|}{6.0616} & \multicolumn{2}{|c|}{9.511} \\
\hline
\end{tabular}

\section{Nonlinear Oscillator}

Sometimes, dynamic problems are governed by $m \ddot{x}+c \dot{x}+k x=f(t)$ having nonlinear stiffness $\left(k_{1} x+k_{2} x^{2}+\cdots\right)$ which result in nonlinear differential equations (nonlinear oscillators). In case of uncertain nonlinear oscillators, the SIVIA Monte Carlo method has been implemented using nonlinear equations obtained based on the Runge-Kutta fourth-order method [4, 8]. As such, the enclosure obtained in the present section yields a validated enclosure rather than the tightest bound. There exist several validated interval methods and solvers in DynIbex [15] and CAPD [5] libraries for obtaining validated bounds.

Example 4 Consider the Van der Pol equation (crisp or precise case given in Akbari et al. [1]),

$$
\ddot{x}(t)+0.15\left(1-x^{2}\right) \dot{x}+1.44 x=0
$$

subject to uncertain initial conditions $x(0) \in[0.1,0.3]$ and $\dot{x}(0)=0$.

The system of the first-order differential equation corresponding to (12) is obtained as 
Table 3 Interval enclosure of $z_{2}$

\begin{tabular}{|c|c|c|c|c|}
\hline \multirow[t]{3}{*}{ Iterations } & \multicolumn{4}{|c|}{ SIVIA (0.001 precision) and Monte Carlo samples } \\
\hline & \multicolumn{2}{|l|}{100,000 samples } & \multicolumn{2}{|l|}{1000 samples } \\
\hline & $\underline{z}_{2} \in$ & $\bar{z}_{2} \in$ & $\underline{z}_{2} \in$ & $\bar{z}_{2} \in$ \\
\hline 1 & {$[0.4565,0.4617]$} & {$[0.6825,0.6889]$} & {$[0.4565,0.4617]$} & {$[0.6825,0.6889]$} \\
\hline 2 & {$[0.4565,0.4581]$} & {$[0.6869,0.6887]$} & {$[0.4565,0.4591]$} & {$[0.6859,0.6887]$} \\
\hline 3 & {$[0.4566,0.4575]$} & {$[0.6872,0.6886]$} & {$[0.4565,0.4579]$} & {$[0.6864,0.6887]$} \\
\hline 4 & - & - & {$[0.4565,0.4577]$} & {$[0.6867,0.6887]$} \\
\hline 5 & - & - & {$[0.4565,0.4575]$} & {$[0.6869,0.6887]$} \\
\hline 6 & - & - & {$[0.4565,0.4574]$} & {$[0.687,0.6889]$} \\
\hline$\left[z_{2}\right]$ & \multicolumn{2}{|c|}{$[0.46,0.69]$} & \multicolumn{2}{|c|}{$[0.46,0.69]$} \\
\hline Time (s) & \multicolumn{2}{|l|}{6.7797} & \multicolumn{2}{|l|}{7.5464} \\
\hline \multirow[t]{2}{*}{ Iterations } & \multicolumn{2}{|l|}{100 samples } & \multicolumn{2}{|l|}{10 samples } \\
\hline & $\underline{z}_{2} \in$ & $\bar{z}_{2} \in$ & $\underline{z}_{2} \in$ & $\bar{z}_{2} \in$ \\
\hline 1 & {$[0.4565,0.4617]$} & {$[0.6825,0.6889]$} & {$[0.3796,0.385]$} & {$[0.5935,0.6007]$} \\
\hline 2 & {$[0.4565,0.4609]$} & {$[0.6826,0.6887]$} & {$[0.4565,0.4609]$} & {$[0.6854,0.6887]$} \\
\hline 3 & {$[0.4565,0.4599]$} & {$[0.6858,0.6887]$} & {$[0.4565,0.4601]$} & {$[0.6828,0.6886]$} \\
\hline 4 & {$[0.4566,0.4578]$} & {$[0.6863,0.6885]$} & {$[0.4565,0.4589]$} & {$[0.6836,0.6886]$} \\
\hline 5 & {$[0.4566,0.4577]$} & {$[0.6864,0.6891]$} & {$[0.4565,0.4584]$} & {$[0.6839,0.6885]$} \\
\hline 6 & {$[0.4566,0.4576]$} & {$[0.6866,0.689]$} & {$[0.4565,0.4581]$} & {$[0.685,0.6885]$} \\
\hline 7 & {$[0.4566,0.4574]$} & {$[0.6867,0.689]$} & {$[0.4565,0.4577]$} & {$[0.6856,0.6885]$} \\
\hline 8 & - & - & {$[0.4565,0.4575]$} & {$[0.686,0.6885]$} \\
\hline 9 & - & - & {$[0.4566,0.4574]$} & {$[0.6865,0.6884]$} \\
\hline$\left[z_{2}\right]$ & \multicolumn{2}{|c|}{$[0.46,0.69]$} & \multicolumn{2}{|c|}{$[0.46,0.69]$} \\
\hline Time (s) & \multicolumn{2}{|c|}{9.2454} & \multicolumn{2}{|c|}{24.847} \\
\hline
\end{tabular}

$$
\begin{aligned}
& \dot{u}=v=f_{u}(t, u, v) \\
& \dot{v}=0.15\left(u^{2}-1\right) v-1.44 u=f_{v}(t, u, v)
\end{aligned}
$$

subject to initial conditions $u(0) \in[0.1,0.3]$ and $v(0)=0$. Using Runge-Kutta fourth-order method, the nonlinear constraints involved in the computation of (12) are

$$
\begin{gathered}
u_{n+1}=u_{n}+\frac{h}{6}\left(k_{1}+2 k_{2}+2 k_{3}+k_{4}\right) \\
v_{n+1}=v_{n}+\frac{h}{6}\left(l_{1}+2 l_{2}+2 l_{3}+l_{4}\right)
\end{gathered}
$$

where

$$
k_{1}=h f_{u}\left(t_{n}, u_{n}, v_{n}\right), l_{1}=h f_{v}\left(t_{n}, u_{n}, v_{n}\right) \text {, }
$$


Table 4 Instantaneous solution enclosure of $\left.x(t)\right|_{t=T}$

\begin{tabular}{l|l|l}
\hline \multirow{2}{*}{$\mathrm{T}$} & \multicolumn{2}{|c}{ Enclosures } \\
\cline { 2 - 3 } & {$[x](T)=[u](T)$} & {$[v](T)$} \\
\hline 0.1 & {$[0.0993,0.2979]$} & {$[-0.0428,-0.0143]$} \\
0.2 & {$[0.0972,0.2915]$} & {$[-0.0844,-0.0281]$} \\
0.3 & {$[0.0937,0.281]$} & {$[-0.1242,-0.0413]$} \\
\hline
\end{tabular}

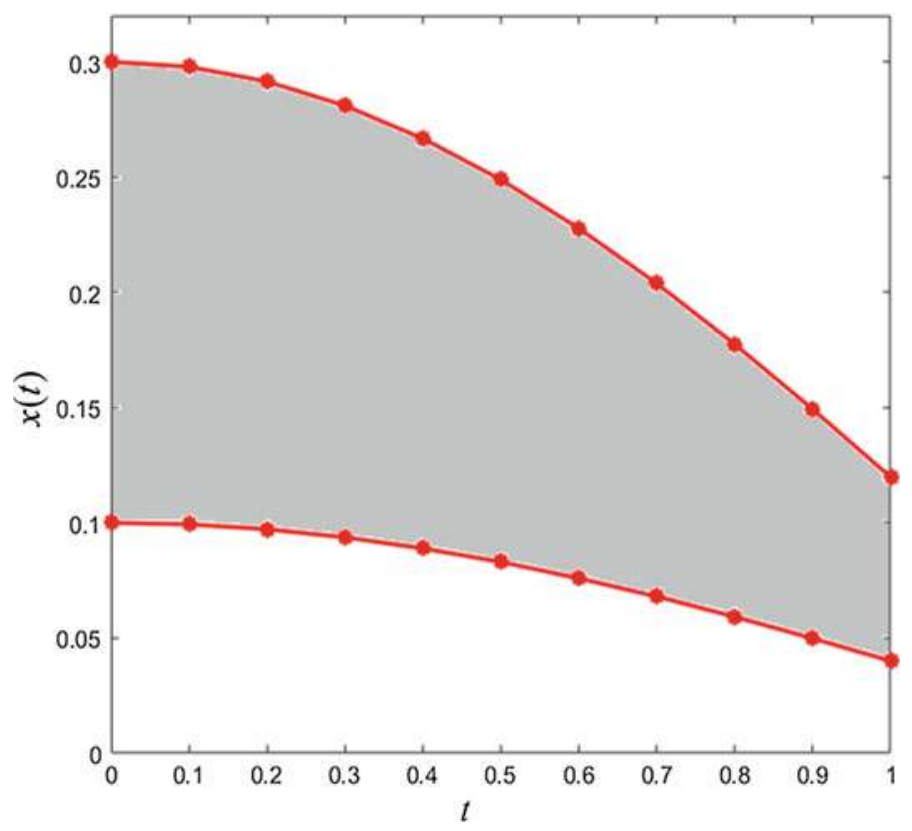

Fig. 5 Enclosure of $x(t)$ for $t \in[0,1]$

$$
\begin{gathered}
k_{2}=h f_{u}\left(t_{n}+\frac{h}{2}, u_{n}+\frac{k_{1}}{2}, v_{n}+\frac{l_{1}}{2}\right), l_{2}=h f_{v}\left(t_{n}+\frac{h}{2}, u_{n}+\frac{k_{1}}{2}, v_{n}+\frac{l_{1}}{2}\right), \\
k_{3}=h f_{u}\left(t_{n}+\frac{h}{2}, u_{n}+\frac{k_{2}}{2}, v_{n}+\frac{l_{2}}{2}\right), l_{3}=h f_{v}\left(t_{n}+\frac{h}{2}, u_{n}+\frac{k_{2}}{2}, v_{n}+\frac{l_{2}}{2}\right), \\
k_{4}=h f_{u}\left(t_{n}+h, u_{n}+k_{3}, v_{n}+l_{3}\right) \text { and } l_{4}=h f_{v}\left(t_{n}+h, u_{n}+k_{3}, v_{n}+l_{3}\right) .
\end{gathered}
$$

Using Algorithm 1 with respect to constraints (13), and (14), the validated enclosure of $\left.x(t)\right|_{t=T}$ is obtained and incorporated in Table 4 and Fig. 5. 


\section{Conclusion}

Generally, dynamical systems occurring in various science and engineering problems are governed by nonlinear equations or nonlinear differential equations. An iterative procedure based on SIVIA and the Monte Carlo method has been proposed for the computation of exact enclosure of nonlinear equations having imprecise or uncertain variables. The effectiveness of the SIVIA Monte Carlo method has also been verified based on the considered test problems that yield exact enclosures even with respect to very few sample values. So, the method may be well implemented in the computation of exact enclosures of various nonlinear equations irrespective of the dependency problem. Further, the method has also been implemented to compute validated enclosure in case of Van der Pol oscillator. Accordingly, the method may be applied to other practical problems governed by nonlinear system of equations or nonlinear differential equations involving uncertain parameters.

\section{Appendix}

Forward-backward contractor: The forward-backward contractor is based on constraint $f(\mathbf{x})=0$ where $\mathbf{x} \in[\mathbf{x}]$ and $[\mathbf{x}] \in \mathbb{\mathbb { R } ^ { n }}$ which is illustrated using an example problem.

Example A1 Perform forward-backward contractor subject to constraint $w=2 u+$ $v$ where $[w]=[3,20],[u]=[-10,5]$, and $[v]=[0,4]$.

Here, the constraint $w=2 u+v$ may be expressed in terms of function $f$ as $f(u, v, w)=w-2 u-v$. Further, the possible different forms of the constraint that may be written are

$$
\begin{aligned}
& u=\frac{w-v}{2} \\
& v=w-2 u \\
& w=2 u+v
\end{aligned}
$$

The forward-backward steps are then followed with respect to classical interval computations mentioned in Sect. 2 as

$$
\begin{gathered}
{[u] \cap\left(\frac{[w]-[v]}{2}\right)=[-10,5] \cap\left(\frac{[3,20]-[0,4]}{2}\right)=[-0.5,5]} \\
{[v] \cap([w]-2[u])=[0,4] \cap([3,20]-2[-0.5,5])=[0,4]} \\
{[w] \cap(2[u]+[v])=[3,20] \cap(2[-0.5,5]+[0,4])=[3,14]}
\end{gathered}
$$


As such, the new interval bounds are $[w]=[3,14],[u]=[-0.5,5]$, and $[v]=[0,4]$.

Fixed-point contractor: A fixed-point contraction associated with $\psi$ is implemented with respect to the constraint $f(\mathbf{x})=0$ as $\mathbf{x}=\psi([\mathbf{x}])$, where $\mathbf{x} \in[\mathbf{x}] \in \mathbb{I} \mathbb{R}^{n}$. The fixed-point contractor with respect to constraint $u^{2}+2 u+1=0$ is performed as

$$
\begin{aligned}
u \in[u] \text { and } u=\psi(u) & \Longrightarrow u \in[u] \text { and } u \in \psi([u]) \\
& \Longrightarrow u \in[u] \cap[\psi]([u])
\end{aligned}
$$

In case of the implementation of forward-backward contractor along with fixedpoint contractor helps in the computation of the forward-backward contractor until the fixed interval is reached.

\section{References}

1. Akbari M, Ganji D, Majidian A, Ahmadi A (2014) Solving nonlinear differential equations of vanderpol, rayleigh and duffing by agm. Front Mech Eng 9(2):177-190

2. Alefeld G, Herzberger J (2012) Introduction to interval computation. Academic Press, London

3. Chabert G, Jaulin L (2009) Contractor programming. Artif Intell 173:1079-1100

4. Chakraverty S, Mahato NR, Karunakar P, Rao TD (2019) Advanced numerical and semianalytical methods for differential equations. Wiley, Hoboken

5. Computer assisted proofs in dynamics group (capd). http://capd.ii.uj.edu.pl/

6. Desrochers B, Pyibex. http://benensta.github.io/pyIbex/sphinx/index.html

7. Dezert J, Han D, Tacnet JM (2017) Multi-criteria decision-making with imprecise scores and BF-TOPSIS. In: Information Fusion (Fusion), 2017 20th International Conference on. pp 1-8. International Society of Information Fusion (ISIF)

8. Gerald CF (2004) Applied numerical analysis. Pearson Education India

9. Hansen ER (1975) A generalized interval arithmetic. In: Interval mathematics. Springer, pp $7-18$

10. Jaulin L, Kieffer M, Didrit O, Walter E (2001) Applied interval analysis: with examples in parameter and state estimation, robust control and robotics, vol 1. Springer, London

11. Krämer W (2006) Generalized intervals and the dependency problem. In: PAMM: proceedings in applied mathematics and mechanics, vol 6. Wiley Online Library, pp 683-684

12. Moore RE (1962) Interval arithmetic and automatic error analysis in digital computing. Ph.D. Dissertation, Department of Mathematics, Stanford University

13. Moore RE (1979) Methods and applications of interval analysis, vol 2. Siam

14. Moore RE, Kearfott RB, Cloud MJ (2009) Introduction to interval analysis. SIAM Publications, Philadelphia, PA

15. Sandretto JAd, Chapoutot A, Mullier O, Dynibex. http://perso.ensta-paristech.fr/ chapoutot/ dynibex/

16. Stolfi J, De Figueiredo L (2003) An introduction to affine arithmetic. Trends Appl Comput Math 4(3):297-312 